\title{
Médiation scientifique
}

Retour sur la genèse d'une catégorie et ses usages

\section{Andrée Bergeron}

\section{(2) OpenEdition}

Journals

Édition électronique

URL : http://journals.openedition.org/aes/876

DOI : $10.4000 /$ aes.876

ISSN : 2258-093X

Éditeur

Laboratoire LISAA

Référence électronique

Andrée Bergeron, « Médiation scientifique », Arts et Savoirs [En ligne], 7| 2016, mis en ligne le 06 décembre 2016, consulté le 19 avril 2019. URL : http://journals.openedition.org/aes/876 ; DOI : 10.4000/aes.876

Ce document a été généré automatiquement le 19 avril 2019.

Centre de recherche LISAA (Littératures SAvoirs et Arts) 


\title{
Médiation scientifique
}

\author{
Retour sur la genèse d'une catégorie et ses usages
}

\section{Andrée Bergeron}

\section{Hériter d'un problème}

1 Dans l'introduction de son ouvrage de référence, Écrire la science, Yves Jeanneret s'attarde longuement sur le malaise récurrent suscité tout au long de son histoire par le terme vulgarisation ${ }^{1}$. Avec ce mot, le français, nous dit-il, a choisi de mettre l'accent sur le peuple; seulement pas n'importe quel peuple : pas le populus, ce peuple qui vote et qui décide, mais le vulgus, le petit peuple, la plèbe ${ }^{2}$.

Il s'agit bien là d'une singularité terminologique française (ou presque ${ }^{3}$ ) puisque d'autres langues témoignent d'autres choix. Ainsi, avec popularization of science ou encore popular science, c'est au populus, le peuple décideur, que la langue anglaise fait référence; d'autres langues latines (l'espagnol divulgación, le portugais divulgação ou l'italien divulgazione) utilisent un terme construit sur le verbe divulguer et dont l'usage n'est pas propre au domaine scientifique mais vaut de manière générale pour désigner le dévoilement d'une chose auparavant secrète - ce faisant, elles insistent sur le secret à briser; la langue allemande quant à elle, avec le mot Verbreitung (Verbreitung des Wissens), insiste sur l'idée d'élargissement (de ceux qui peuvent ainsi accéder à la parole scientifique).

3 Tout au long des deux derniers siècles, la singularité terminologique française n'a cessé de poser problème dans sa société même. Le mot « vulgarisation » est pourtant le résultat d'un choix puisque les deux termes «popularisation » et «vulgarisation » ont un temps coexisté avant qu'au début $\mathrm{du} \mathrm{xx}^{\mathrm{e}}$ siècle l'usage ne consacre ce dernier. Les locuteurs du français ont choisi ce terme, dont l'usage est spécifique à la science (c'est-à-dire en grande partie limité au sens de "vulgarisation scientifique»), et en même temps n'ont cessé de le rejeter à cause de sa proximité avec le mot « vulgaire » et ses dérivés ${ }^{4}$ qui lui confère une connotation négative. 
C'est sans doute l'une des raisons pour lesquelles l'histoire de la vulgarisation est jalonnée de tentatives de remplacement du mot (sinon de la chose) ou, à tout le moins, de sa qualification. On parle ainsi durant l'entre-deux-guerres de «revues de haute vulgarisation $»^{5}$ (celles qui justement ne s'adressent pas au bas peuple mais au spécialiste ou au minimum à l'homme éduqué) tandis que la seconde partie du $\mathrm{xx}^{\mathrm{e}}$ siècle verra naître de nombreux essais de re-nomination, comme :

- «propagande » dans l'immédiat après-guerre,

- « information scientifique », durant les années 1960,

- «communication scientifique », durant les années 1970-1980,

- (diffusion ou promotion de la) « culture scientifique », durant les années 1980-1990

- et désormais «médiation des sciences ».

Quelque chose existe ainsi dont nous ne sommes jamais certains du nom (puisqu'il est périodiquement remis en cause) et qui est néanmoins au cœur des pratiques de professionnels, au cœur de l'action publique ou encore de la réflexion des acteurs ou des chercheurs. Deux questions en découlent.

6 Tout d'abord, ces changements de terminologie doivent-ils être considérés comme correspondant à des catégories d'action distinctes - pour le dire autrement, se traduisentils par de réels changements dans les pratiques? - et alors, en quoi diffèrent-elles? Certes, à chaque époque les discours d'acteurs revendiquent l'évolution en cours comme marquant sinon une rupture du moins une transformation dans les modes et les finalités des actions. Mais les inflexions dans les discours ne suffisent pas toujours à transformer les réalisations effectives.

7 Corollairement, le chercheur (qu'il soit historien, sociologue, spécialiste des sciences de la communication, etc.) peut-il reprendre à son compte, sans plus de problématisation, cette terminologie en tant catégorie d'analyse? Le problème se pose tout particulièrement dans le cas de l'analyse historique qui, sur une durée suffisamment longue, se voit confrontée à ces mutations de vocabulaire, dont elle ne peut que prendre acte et noter que l'on entend ainsi marquer des évolutions. La question reste cependant entière de comprendre quelles ont été ces évolutions (et d'abord, si elles existent) et alors où elles se situent - ce n'est peut-être pas là où les mots l'indiquent à première vue.

\section{Détour onomastique : signaler et cacher}

8 Nommer (des objets, des phénomènes, des personnages) n'est jamais un acte neutre. Cela désigne, signale, voire fait advenir. En littérature, la question du nom revêt pour certains auteurs une importance cruciale. Il en est ainsi de Georges Perec, dont l'œuvre renferme de nombreux cas d'« instabilité du nom propre "*. Quel petit vélo au guidon chromé au fond de la cour ${ }^{7}$, pousse à l'extrême la nomination instable. Celui qui paraît en être le personnage central (et dont il s'agit d'empêcher le départ pour combattre en Algérie) change en effet de nom tout au long de l'ouvrage: Karamanlis, Karawasch, Karawo, Karacouvé, Karaplasm... bref, Karatruc. Comme bien souvent chez Perec le jeu (ici avec le nom) n'est pas gratuit et l'apparente légèreté cache des choses plus profondes ${ }^{8}$ : plus encore que la guerre pour laquelle l'homme sans nom refuse de partir lui préférant de loin les bras de sa belle - la vie plutôt que la mort -, c'est son histoire familiale que Georges Perec encrypte au passage dès son deuxième livre ${ }^{9}$. On peut encore remarquer une autre particularité de ce texte : contrairement aux apparences, Karamachin n'est sans 
doute pas à proprement parler le héros, le personnage central de l'histoire. Ce livre marquait, abruptement pour les lecteurs séduits par Les Choses $^{10}$ la première mise en œuvre d'une facette de l'écriture perecquienne, plus formaliste et plus ludique ${ }^{11}$. Et en effet, au travers des figures de rhétorique, «figures centrales du récit, les véritables héroïnes en quelque sorte", c'est "la littérature elle-même et le pouvoir qu'elle croit avoir de nommer, de structurer comme de déstructurer ou de détruire » qui parait bien être l'objet central de cette " épopée $»^{12}$.

Sur ces deux aspects, un parallèle peut être fait avec la vulgarisation.

10 Les changements répétés de dénomination, symptômes du malaise persistant entourant l'activité vulgarisatrice, ne sont pas gratuits eux non plus. Ils ne sont pas seulement le résultat de tentatives d'euphémisation visant à fuir un mot trop proche de "vulgaire » et de ses dérivés. En effet, les appellations (temporairement) retenues ne sont choisies au hasard; aucune d'entre elles n'est neutre et chacune doit être mise en regard d'une configuration historique, sociale et épistémique particulière :

- au sortir de la seconde guerre mondiale, il s'agit de reconstruire la nation et cela passe, pensent les responsables, par la formation de personnels scientifiques et techniques à tous les niveaux et en nombre suffisant - et pour cela il faut convaincre des candidats, en usant s'il le faut de moyens que l'on n'appelle pas encore publicitaires (propagande);

- dans les années 1960, les débuts de l'informatisation de la société font de la gestion et de l'accès à l'information un enjeu primordial - et c'est sous cet angle d'abord (permettre au plus grand nombre d'accéder à l'information scientifique) que sont perçues en France, par les pouvoirs publics comme par les responsables des institutions concernées, les questions de vulgarisation;

- au cours des années 1970, la communication monte en puissance tandis que les enjeux communicationnels autour des sciences et des techniques se font d'autant plus forts que la contestation qui marque ces années-là touche de plein fouet les sciences et les technologies, surtout lorsqu'elles sont liées au nucléaire ${ }^{13}$.

11 Finalement, la vulgarisation, au travers de ces changements terminologiques, n'est peutêtre pas davantage le principal personnage de notre histoire que Karachose ne l'était du Petit vélo. L'inventivité lexicographique n'a peut-être pas tant pour fonction, contrairement à ce que les discours d'acteurs semblent indiquer, d'attester de changements dans les registres d'action des vulgarisateurs que de prendre acte de changements dans les sciences, dans les sociétés, et donc dans leur articulation et dans le rôle dévolu aux sciences à chaque période.

12 C'est ce que la seconde partie de ce texte souhaite analyser en revenant sur l'émergence de l'expression la plus récente, celle dont l'usage est désormais répandu: «médiation scientifique ».

13 Plusieurs pistes peuvent être explorées pour retracer la genèse de l'expression médiation scientifique. J'en distinguerai ici successivement trois.

\section{La médiation culturelle : œuvrer à la professionnalisation}

14 La première renvoie à la médiation culturelle. Ce que l'on appelle ainsi se développe dans le secteur culturel à partir de la seconde partie des années 1980 et surtout au cours des années 1990. La fin des années 1990 et le début des années 2000 verront sa consécration. 
L'idée n'est pas entièrement nouvelle puisque l'objectif en est de partager des œuvres et des savoirs avec le plus grand nombre. Héritière de l'éducation populaire, des méthodes de pédagogie active et de l'action culturelle des années 1960-1970, la médiation culturelle s'en démarque pourtant sur un point important : elle inverse le sens du mouvement. En effet, si les premières partaient des gens pour aller vers les œuvres, la seconde (dans une première période au moins) met en avant les œuvres et les savoirs qu'il s'agit d'adresser à des gens ${ }^{14}$.

Autre élément de contexte qui a son importance, la notion de médiation culturelle monte en puissance en même temps que se développent (notamment à la faveur des contrats aidés pour l'emploi des jeunes) des fonctions de "médiateurs culturels ", que ce soit dans les institutions culturelles elles-mêmes ou dans le cadre des politiques de la ville. À cette même époque (fin des années 1980), parmi d'autres initiatives de formation aux métiers de la culture, naissent dans les universités de Paris 3 et Paris 8 les premières formations à la médiation culturelle ${ }^{15}$. Mais c'est surtout à partir de 1997, avec la mise en place des emplois-jeunes, que la question de la reconnaissance de ce champ d'activité comme champ professionnel en propre se pose avec acuité.

En 1995, paraît l'ouvrage d'élisabeth Caillet, À l'approche du musée, la médiation culturelle. L'auteur y défend la nécessité de cette (encore) nouvelle fonction et en resitue l'émergence ${ }^{16}$, notamment dans le droit fil de ce qui s'est développé au Québec sous l'intitulé d'« interprétation». Grande promotrice de la médiation culturelle, Élisabeth Caillet en est à plus d'un titre l'une des instigatrices majeures en France : elle a travaillé aux actions d'éducation et de formation de la Cité des Sciences (nous y reviendrons) dans les premiers et avant-premiers temps de celle-ci, est à l'origine de la formation à la médiation culturelle de Paris 8 déjà mentionnée, puis a occupé différentes fonctions au ministère de la culture avant de créer au CNED le campus numérique pour la formation des médiateurs culturels, CampusCultura.

En 2002, la loi sur les musées de France ${ }^{17}$ va encore contribuer à légitimer l'idée de médiation culturelle puisque son article 7 subordonne l'obtention du label Musée de France à l'existence "d'un service ayant en charge les actions d'accueil des publics, de diffusion, d'animation et de médiation culturelles » et surtout précise que « ces actions sont assurées par des personnels qualifiés. »

La notion de médiation scientifique va partiellement se construire sur celle de médiation culturelle et sera, elle aussi, fortement ancrée dans une problématique de reconnaissance professionnelle des personnels assurant cette fonction. En 1998 en effet, le ministère de l'enseignement supérieur engage la rénovation de son référentiel de branche d'activité professionnelle pour les emplois ITRF (REFérentiel des Emplois-types de la Recherche et de l'ENseignement Supérieur [REFERENS]). Parmi les établissements-pilotes qui aident à l'élaboration du référentiel rénové, figure le Palais de la découverte.

Une grande partie des emplois de ce dernier organisme est alors occupée par ceux que l'on appelle encore des "chargés d'exposés ", c'est-à-dire des personnes assurant des fonctions de médiation en face-à-face auprès des visiteurs. Non sans discussion, l'appellation "médiateur scientifique » est adoptée pour qualifier ces fonctions, l'un des objectifs du groupe de travail constitué pour l'occasion étant justement de permettre la comparaison avec des fonctions similaires dans d'autres champs disciplinaires. La proposition sera retenue par le ministère et un métier de médiateur scientifique (avec tous les grades permettant une évolution de carrière) fera son apparition quelques mois 
plus tard dans le référentiel REFERENS. Malgré les oppositions parfois fortes ${ }^{18} \mathrm{du}$ début la dénomination sera reprise et très rapidement revendiquée par les personnels du Palais eux-mêmes. Tout laisse à penser qu'elle répondait à des besoins : nommer un métier avec ses compétences propres, mais encore valoriser le savoir-faire d'un établissement qui n'avait pas, contrairement à ses homologues parmi les «musées de l'Éducation nationale », fait l'objet d'une rénovation d'envergure en insistant sur ce qu'il revendique comme faisant sa force et son identité : la médiation humaine.

Au-delà du Palais de la découverte, l'adoption du terme a eu pour effet de faire exister dans la fonction publique, via son référentiel des métiers, des métiers de «médiateurs scientifiques ». Le changement terminologique fera par la suite son apparition dans les intitulés de formations universitaires: à l'université Louis Pasteur de Strasbourg, par exemple, où un parcours (professionnel) « Patrimoine et médiation » a été mis en place à la fin des années 2000 dans le cadre d'une formation de master Science, technologie et société, elle-même intitulée « Épistémologie et médiation scientifique ».

Un point mérite d'être souligné qui traverse toute cette chronologie brossée à gros trait : la montée en puissance de la notion de médiation appliquée au secteur de la culture (et dans un deuxième temps de la culture scientifique) est constamment liée à des questions de professionnalisation, qu'il s'agisse de reconnaître et valoriser les savoir-faire de professionnels en activité (quel que soit leur statut) ou de former les (futurs) professionnels des institutions culturelles, qu'elles soient ou non scientifiques.

\section{Rompre avec l'ancien, signaler le moderne}

Tandis que tout ceci se déroulait, l'expression «médiation scientifique » était en usage, relativement fréquent mais encore confiné, dans une institution d'importance : la Cité des sciences et de l'industrie qui ouvre ses portes en 1986 mais affiche déjà plusieurs années de fonctionnement consacrées à sa conception et à sa réalisation. On y parle très tôt, dès l'ouverture voire avant, de médiation scientifique alors que ce terme ne semble pas d'usage courant dans d'autres contextes. Les recherches faites sur l'occurrence «médiation scientifique» dans les bases de données bibliographiques jusqu'à la fin des années 1990 en témoignent : elles conduisent en effet très majoritairement à des écrits émanant de professionnels ou anciens professionnels de la Cité des Sciences.

Plusieurs raisons peuvent être invoquées. Tout d'abord, l'institution, à l'instar de ses homologues plus anciennes en leur temps ${ }^{19}$, se veut résolument nouvelle. Il ne s'agit donc pas d'y faire de la "vulgarisation » mais quelque chose qui s'en démarquerait, ce que l'usage d'une nouvelle terminologie permet d'indiquer. Ensuite, les groupes de travail qui président à sa conception intègrent un certain nombre de personnalités qui militent pour « un autre traitement culturel » (mot alors plus usité) des sciences : Élisabeth Caillet déjà mentionnée, mais encore des personnalités comme les membres du groupe Scientifiction (Françoise Bastide, Denis Guedj, Bruno Latour et Isabelle Stengers) ${ }^{20}$ dont certains travaillent au même moment à la mise en place d'une nouvelle sociologie des sciences qui considère moins ces dernières comme productrices d'un discours de vérité sur le monde que comme un mode de description d'un monde constitué de collectifs et d'associations liés entre eux par des attachements et, justement, par des médiations. Le mot médiation permet ainsi de faire implicitement d'une pierre deux coups : non seulement il indique de nouvelles formes d'adresse au public (dans le droit fil de la tradition d'éternelle rupture 
des institutions muséales scientifiques), mais aussi il renvoie à un cadre d'analyse renouvelé (et encore jugé iconoclaste) des sciences elles-mêmes.

L'ouverture de la Cité des sciences en 1986, une fois encore, a été perçue comme susceptible de générer un bassin d'emplois pour de jeunes professionnels. Il s'agit alors de les former. C'est ce que pense, avec d'autres, Denise Devèze-Berthet, professeur en sciences de l'information et de la communication à l'université Paris 7 :

Sous la tutelle de l'Éducation nationale, le Palais [de la découverte] a fonctionné avec un corps de pédagogues détenteurs du savoir institué. L'histoire récente de la Cité [des sciences] est au contraire marquée par le repli des médiateurs issus de la communauté scientifique au profit des professionnels de la communication et du marketing. Vitrine de l'innovation, instrument de valorisation de l'État, produit de consommation culturelle, telles sont les caractéristiques de ce musée gigantesque. C'est en spéculant sur ses capacités d'embauche que se sont préparés les premiers projets de formation à la médiation des sciences et des techniques. ${ }^{21}$

On voit ici apparaître un deuxième élément important. La médiation des sciences telle qu'elle se pratique à la Cité des sciences (et telle qu'elle se pratiquera ensuite) se pose en rupture, plus ou moins nette, sur un point supplémentaire : elle n'est plus d'abord le fait de "médiateurs issus de la communauté scientifique», elle est désormais le fait de professionnels qui peuvent être issus d'autres disciplines. C'est ainsi une professionnalisation plus autonome, ne se légitimant plus au seul nom de la science, qui apparaît avec la médiation scientifique. De nouveau, médiation et professionnalisation avancent de conserve.

Présent sur les questions de formation, le monde académique l'est aussi pour ce qui concerne la recherche. Parmi les chercheurs travaillant sur les politiques culturelles ou sur les musées et les expositions, nombreux sont ceux qui s'inscrivent dans le champ des sciences de l'information et de la communication. Les recherches se font fréquemment en très proche connexion avec les institutions culturelles, quand ces dernières n'en sont pas elles-mêmes les commanditaires. C'est tout particulièrement le cas en ce qui concerne les institutions culturelles scientifiques (les musées, par exemple). Ces collaborations sont soutenues par une série d'initiatives des ministères concernés, dont le programme interministériel REMUS d'aide à la recherche en muséologie des sciences et des techniques lancé en 1989 constitue l'un des meilleurs exemples ${ }^{22}$ (REMUS, 1995).

La forte présence des sciences de l'information et de la communication permet sans doute partiellement de comprendre pourquoi, bien que la médiation culturelle se soit construite en France en s'inspirant du modèle d'«interprétation» québécois ${ }^{23}$, ce n'est pas ce dernier terme qui a été retenu mais un mot («médiation») étymologiquement proche d'un concept central pour ce champ de recherche («media »).

Ceci n'est pas sans conséquence. En effet, si les catégories «médiation » et «médiation scientifique » étaient dans tout ce qui précède essentiellement des catégories d'action (pragmatiques, lorsqu'il s'agit de désigner ce qui se fait ; programmatiques, pour ce qu'il s'agirait de faire) ou des catégories administratives (lorsqu'il s'agit de nommer une fonction dans un établissement public, de rendre obligatoire un ensemble d'actions dans un musée, d'habiliter des formations universitaires), lorsqu'elles sont reprises dans les travaux des chercheurs elles deviennent d'emblée des catégories d'analyse. La question se pose alors du cadre conceptuel dans lequel ces catégories ont été forgées et de sa validité lors de leur transfert dans d'autres champs épistémiques comme la littérature ou l'histoire (des sciences ou culturelle). Si rien n'autorise à disqualifier de prime abord de tels transferts, un exercice de réflexivité paraît néanmoins salutaire. 


\section{L'important c'est de participer ou playing the notes but missing the music}

L'essor du concept de médiation à cette période ne se limite ni aux sciences, ni même au secteur culturel. Les années 1980, parfois appelées « décennie de la médiation $»^{24}$, et les années 1990 correspondent à un moment de montée en puissance de la médiation qu'il s'agisse de questions publiques ou privées. Médiation familiale, médiation d'entreprise (Médiateur de la SNCF, par exemple), médiation d'affaire, médiation d'entreprise de presse $^{25}$, Haut-Conseil de la médiation, Médiateur de la république, Médiateur des enfants, Médiateur du cinéma, médiateurs sociaux : les médiations sont partout.

Même si l'expression « médiation scientifique » recouvre désormais un sens spécifique, il n'est pas inintéressant de resituer l'avènement de cette médiation-ci dans ce contexte global-là et de la confronter aux conclusions auxquelles aboutissent les chercheurs qui considèrent la question des médiations dans la diversité de leurs usages (par exemple, Briant et Palau, 19996). Deux points semblent particulièrement pertinents pour qui s'intéresse aux articulations entre sciences et sociétés. Tout d'abord, aux yeux des sciences sociales, la médiation apparaît essentiellement comme un mécanisme de régulation sociale, dont la montée contemporaine doit être mise en regard avec celle des nombreux dispositifs de soft-law et dont la neutralité supposée est plus que questionnable. Ensuite, l'explosion de la médiation se passe à un moment de l'évolution de nos sociétés qui voit une mutation profonde du mode d'insertion de l'individu dans la société et la recherche croissante d'évitement du conflit.

Qu'en est-il dans le même temps de l'évolution des relations entre sciences et société ? Du côté du débat académique comme, dans un second temps, de la gestion politique des sciences, les années 1990 et celles qui suivent sont marquées par la promotion croissante de la participation. Dans la production savante internationale, le paradigme de Public understanding of science (dominant dans les années 1990 et qui attribuait à un manque de compréhension des énoncés et pratiques scientifiques par le public la responsabilité d'un discrédit des sciences et des techniques et de leur contestation croissante) est progressivement remis en question au profit de la promotion du Public engagement in/with science $e^{27}$ (engagement « actif » des publics dans les sciences), plus en amont du processus de recherche. Les pouvoirs publics, en Grande-Bretagne d'abord puis très vite via l'Union Européenne dans l'ensemble des pays européens, reprendront ce credo et l'inscriront à l'agenda des institutions et des grands programmes scientifiques. À l'instar de la médiation vis-à-vis de la vulgarisation, le Public engagement with science revendique une rupture nette avec les anciennes pratiques. Il ne s'agit plus désormais de combler le déficit de connaissance des publics, mais de reconnaître des compétences distribuées et, moyennant la mise en place de technologies sociales ad-hoc, d'affirmer l'introduction d'une dimension consultative, voire participative, au processus de production des connaissances. Pourtant, certains auteurs, comme Brian Wynne ${ }^{28}$, s'interrogent sur la traduction concrète de ce changement de paradigme. Les cadrages imposés par les dispositifs de consultation, la formulation des questions ou encore le statut accordé aux résultats des consultations du public jouent un rôle considérable quant à la nature finale de l'ensemble du processus. Certes, des publics ou leurs représentants ont désormais un droit de parole mais quel est le statut accordé aux conclusions de ces consultations? Considérant notamment que les options proposées par ces dispositifs sont très restreintes 
(refus ou acceptation en bloc) et ne permettent ni de construire collectivement des alternatives, ni de négocier les conditions qui rendraient une technologie acceptable ${ }^{29}$, Brian Wynne émet un constat pessimiste : si les évolutions dans les discours sont notables et admettent aujourd'hui la légitimité de la parole sociale sur les choix technoscientifiques, la traduction pratique de ces discours est plus modeste - on joue bien les notes de la nouvelle chanson, mais sans en restituer la mélodie.

L'essor de la médiation scientifique s'inscrit également dans le mouvement qui vient d'être sommairement décrit et qui promeut un engagement plus « actif » des publics, des visiteurs, des citoyens.

On peut remarquer pour conclure que toute cette évolution conduit vers ce qui ressemble à un paradoxe.

Durant les années 1970, des analyses critiques de la vulgarisation scientifiques et de son rôle social avaient été produites. L'un des principaux apports de ces travaux avait été la remise en cause du prétendu fossé séparant savants et ignorants et que la vulgarisation s'était donné comme mission de combler ${ }^{30}$. L'existence d'un tel fossé y apparaissait fortement sujette à caution et sa principale utilité semblait d'abord être de légitimer la mission vulgarisatrice, le travail du "troisième homme», celui qui se donne comme mission de favoriser la communication entre savants et ignorants.

Deux décennies plus tard, émerge la figure du médiateur scientifique. Celle-ci revendique sa nouveauté et fonde partiellement sa légitimité sur son refus de pratiquer une vulgarisation au sens traditionnel, en s'appuyant pour cela sur les analyses critiques antérieures.

Mais finalement, en quoi le médiateur scientifique diffère-t-il fondamentalement du troisième homme si la médiation se définit comme une «action de mise en relation de deux êtres par un troisième " ? Certes, les actions de médiation revendiquent des formes différentes, affirment une égalité entre les parties qu'il s'agit de mettre en présence, assument une responsabilité partagée dans le dysfonctionnement relationnel. Et effectivement, l'inventivité formelle des dispositifs est grande et conduit à une multitude de créations (ou de réinterprétations) des formes d'interaction. Mais la question posée par Brian Wynne garde ici aussi toute sa pertinence : s'agit-il vraiment d'un changement de paradigme ou seulement d'un changement dans les discours?

\section{NOTES}

1. Yves, Jeanneret, Écrire la Science, Paris, Presses universitaires de France, 1994, p. 11-20.

2. Ibid., p. 11.

3. En effet, la langue roumaine utilise la même forme (vulgarizare ştiinţifică) que le français.

4. Ibid., p. 14-15. L'auteur fait par ailleurs remarquer que lorsque la langue anglaise utilise le terme vulgarization, c'est pour désigner de la "mauvaise" popularization. 
5. C'est le cas, par exemple, de la Revue scientifique et de la Revue générale des sciences pures et appliquées étudiées par Virginie Fonteneau et Hélène Gispert, (cité par Delphine, Benoît, «Un malade qui s'ignore, un médecin qui guérit. Les représentations de la médecine dans les Revues de l'Entre-deux-guerres ", thèse de doctorat, Orsay, université Paris-Sud, 2014, p. 48).

6. Pour reprendre une expression utilisée par Bernard Magné (1999) ; voir aussi David Bellos, Georges Perec. Une vie dans les mots, Paris, Le Seuil, 1994, p. 23-28.

7. Georges, Perec, Quel petit vélo à guidon chromé au fond de la cour, Paris, Denoë, 1966.

8. On peut renvoyer aux analyses de Claude Burgelin (Les parties de dominos chez Monsieur Lefevre, Perec avec Freud, Perec contre Freud, Saulxures, Circé, 1996) ou à cette remarque de Jean-Bertrand Pontalis qui fut son analyste: «Perec est l'auteur de La Disparition, un roman dont l'e muet est invisible et le lecteur ne voit que cette voyelle absente. Ce livre n'est pas l'exploit oulipien que bien des critiques ont salué. Il cache en même temps qu'il le révèle un manque incomblable. Il est le signe d'une détresse qui ne peut pas se dire et seulement se deviner. ", Jean-Bertrand Pontalis, Oublieuse mémoire, Le lieu de l'archive, supplément à la Lettre de l'IMEC, J.-B. Pontalis/IMEC, 2011, p. 18.

9. Magné, Bernard, Georges Perec, Paris, Nathan, 1999, p. 103-104.

10. David Bellos, op. cit., p. 348.

11. Sylvie Rosienski-Pellerin, « Jeux péritextuels : "Quel petit vélo à guidon chromé au fond de la cour" de Georges Perec ", Études littéraires, n²3, vol. 1, 1990, p. 27-41, p. 27.

12. Claude Burgelin, Georges Perec, Paris, Le Seuil, 1988, p. 55-57.

13. Andrée Bergeron, "From Databases to "Information for the General Public": the Long Path toward the Emergence of a Public Action for Scientific Popularization in France at the Turn of the 70's », in M. Le Roux (dir.), Communicating Science, Bruxelles, P.I.E. Peter Lang, à paraître.

14. Serge Chaumier et François Mairesse, La médiation culturelle, Paris, Armand Colin, 2013, p. 108-109.

15. Ibid., p. 167. Notons qu'à Paris 7, Jean Duvignaud refuse cette appellation et maintient l'intitulé «formation à l'action culturelle et sociale ».

16. Voir surtout le chapitre I, p. 25-152.

17. Loi $\mathrm{n}^{\circ}$ 2002-5 du 4 janvier 2002 relative aux musées de France publiée au Journal Officiel de le République Française du 5 janvier 2002.

18. Ces oppositions dérivaient en grande partie d'une des acceptions du mot "médiation » renvoyant à la résolution d'un conflit. De quel conflit pouvait-il donc s'agir? Nous reviendrons plus loin sur la pluralité d'usages du mot « médiation ».

19. C'est un classique des grandes institutions muséales scientifiques que de revendiquer leur radicale nouveauté, voire de se poser en anti-musée. C'était le cas du Palais de la découverte en 1937 Jacqueline Eidelman, «Politique de la science ou politique de l'esprit ? Genèse du Palais de la découverte ", Cahiers d'histoire et de philosophie des sciences, n²4, 1988, p. 53-69; Pascal Ory, «Une cathédrale pour les temps nouveaux? le Palais de la découverte (1934-1940) » in R. Robin (dir.), Masses et culture de masse dans les années 30, Paris, les Éditions ouvrières, 1991, p. 180-204) comme, déjà, du Science Museum au tournant $d u x^{e}$ siècle (Robert Bud, «Infected by the Bacillus of Science: The Explosion of South Kensington », in P. J.T. Morris (dir.), Science for the Nation. Perspectives on the History of the Science Museum, Londres, Palgrave Macmillan, 2010, p. 11-40)

20. Sur Scientifiction voir Élisabeth Caillet (avec la collaboration d'Évelyne Lehalle), À l'approche du musée, la médiation culturelle, Lyon, Presses Universitaires de Lyon, 1995, p. 45-47.

21. Denise Devèze-Berthet, "Pourquoi et comment sont nées les formations à la médiation scientifique ", Brise, Bulletin de recherche sur l'information en sciences économiques et sociales, $\mathrm{n}^{\circ} 14$, 1989, p. 115-120. On doit à Denise Devèze-Berthet la mise en place, en 1984, de la première formation de second cycle universitaire en France spécifique à l'« information et communication 
scientifique et technique ", même si dans cet article paru en 1989 elle emploie une autre terminologie.

22. REMUS, La muséologie des sciences et des techniques, Actes du colloque des 12 et 13 décembre 1991, Dijon, OCIM, 1993.

23. Élisabeth Caillet, op. cit.

24. Jean-François Six, Le temps des médiateurs, Paris, Le Seuil, 1990.

25. Jean-Baptiste Legavre, "Les "règles" du médiateur du Monde ou la mise en scène d'une écriture de presse ", Questions de communication, 12, 2007, p. 311-334.

26. Vincent de Briant et Yves Palau, La médiation. Définition, pratiques et perspectives, Paris, Nathan, 1999.

27. Pour une analyse de cette évolution voir W. Michael Bauer, Nick Allum et Steve Miller, «What have we learnt from 25 years of PUS research-liberating and widening the agenda ", Public Understanding of Science, 16 (1), 2007, p. 79-95.

28. Brian Wynne, «Public engagement as a means of restoring public trust in science - hitting the notes, but missing the music? », Community genetics, 9 (3), 2006, p. 211-220.

29. "The only social options left for this supposedly enlightened and rational framework are the binary opposites, of indiscriminate acceptance, or refusal. There is no room left for constructive negotiation of possible alternatives, multiple trajectories, and different technologies, including of different social ends. Nor is there room for negotiation of the proper conditions under which an otherwise unacceptable technology might be acceptable - i.e. which needs to be ensured consistently in practice. ", Ibid, p. 218.

30. Pour une description synthétique de ces travaux et de leur impact sur la façon dont on pense aujourd'hui ces questions voir Bernadette Bensaude-Vincent, "Splendeur et décadence de la vulgarisation scientifique », Questions de communication, 17, 2010, p. 19-32.

\section{RÉSUMÉS}

Cet article s'intéresse à la «médiation scientifique » considérée comme catégorie. Il s'attache à en restituer l'émergence au cours des deux dernières décennies du $\mathrm{xx}^{\mathrm{e}}$ siècle en France dans le double contexte de la montée en puissance des médiations de tous ordres, d'une part, et des constantes tentatives de remplacement du mot "vulgarisation». Il montre les liens forts qui existent entre la promotion de cette nouvelle appellation et la professionnalisation croissante des institutions culturelles (scientifiques ou non).

\section{INDEX}

Mots-clés : vulgarisation scientifique, médiation, médiation scientifique, professionnalisation, soft-law

Keywords : popularization of science, public understanding of science, public engagement with science, professionalization, soft law 
AUTEUR

ANDRÉE BERGERON

Universcience et Centre Alexandre Koyré (EHESS/CNRS/MNHN)- PSL* Research University 